\title{
The curvature of the QCD critical line from analytic continuation
}

\author{
Claudio Bonati \\ Dipartimento di Fisica, Università di Pisa and INFN, Largo Pontecorvo 2, I-56127 Pisa, Italy \\ E-mail: bonatiedf.unipi.it
}

Massimo D'Elia

Dipartimento di Fisica, Università di Pisa and INFN, Largo Pontecorvo 2, I-56127 Pisa, Italy E-mail: delia@df.unipi.it

\section{Marco Mariti}

Dipartimento di Fisica, Università di Pisa and INFN, Largo Pontecorvo 2, I-56127 Pisa, Italy E-mail: mariti@df.unipi.it

\section{Michele Mesiti*}

Dipartimento di Fisica, Università di Pisa and INFN, Largo Pontecorvo 2, I-56127 Pisa, Italy E-mail: michele.mesiti@pi.infn.it

\section{Francesco Negro}

Dipartimento di Fisica, Università di Pisa and INFN, Largo Pontecorvo 2, I-56127 Pisa, Italy E-mail: fnegro@ge.infn.it

\section{Francesco Sanfilippo}

School of Physics and Astronomy, University of Southampton, Southampton SO17 1BJ, United Kingdom

E-mail: f.sanfilippo@soton.ac.uk

\begin{abstract}
We determine the curvature of the pseudo-critical line of strong interactions, coping with the sign problem by the method of analytic continuation from an imaginary chemical potential. We consider $N_{f}=2+1$ stout improved staggered fermions and the tree level Symanzik gauge action, studying $N_{t}=6$ and $N_{t}=8$ lattices for physical quark masses. The pseudocritical temperatures are evaluated by looking at the $T$ dependence of the renormalized chiral condensate and of the renormalized chiral susceptibility. The determinations of the curvature obtained from the two quantities are in good agreement. Moreover, we investigate the effect of including a non-zero strange quark chemical potential and compare our results to previous determinations in the literature.
\end{abstract}

The 32nd International Symposium on Lattice Field Theory,

23-28 June, 2014

Columbia University New York, NY

\footnotetext{
* Speaker.
} 


\section{The critical line of QCD}

The fact that strongly interacting matter undergoes a transition at finite temperature, where chiral symmetry is restored and quark and gluons are not confined, is a well established property of QCD. In this regard, Lattice QCD simulations yield evidence for a smooth crossover, instead of a proper phase transition ${ }^{1}$, to take place at a temperature $T_{c}$ around $155 \mathrm{MeV}$ at zero baryon density $[1,2]$.

It is of the utmost importance to determine what is the effect of a finite baryon chemical potential $\mu_{B}$ on the critical temperature, in order to obtain reliable QCD predictions to be used in phenomenologically relevant contexts, like e.g. the physics of the early Universe, the description of neutron stars and the physics of heavy ion collisions.

Unfortunately, it is currently impossible to determine directly this dependence by means of lattice simulations, because of the infamous sign problem. Nonetheless, many approaches have been devised to partially circumvent this issue, for example the reweighting method, the Taylor expansion method, the canonical approach and the method of analytic continuation from an imaginary chemical potential [3]. Hybrid approaches have also been conceived [4]. The goal of these strategies is to obtain reliable predictions from the lattice, for at least small values of the $\mu / T$ ratio. In this regime, an analytic parametrization of $T_{C}$ as a function of $\mu_{B}$ at lowest order is the following:

$$
\frac{T\left(\mu_{B}\right)}{T_{c}}=1-\kappa\left(\frac{\mu_{B}}{T\left(\mu_{B}\right)}\right)^{2}+O\left(\mu_{B}^{4}\right)
$$

where $\kappa$ is the curvature and $T_{c}$ is the critical temperature at zero baryon chemical potential. The linear term is forbidden because of the QCD symmetry under charge conjugation.

The critical line in the QCD phase diagram can be compared to the so called chemical freeze-out curve in the $T-\mu_{B}$ plane, which is determined in heavy ion collision experiments. By looking at the abundances of the yields of collisions, it is indeed possible to locate the point of last chemical and thermal equilibrium in the phase diagram, according to a thermal-statistical model [5]. The collection of these points in the phase diagram represents the chemical freeze-out curve. In general one can only assume that chemical equilibrium is reached after hadronization, but it is nonetheless sensible to suppose that this happens shortly after, which implies that the chemical freeze-out curve should lie just below the critical line.

Actually, there is not a good agreement between lattice determinations of $T_{c}$ and the chemical freeze-out estimate. For example, lattice results yield a value of $\kappa$ which is smaller by a factor 2-3 than the one obtained from the freeze-out curve [6], even if a recent improved reanalysis of available experimental data yields a reduction of the discrepancy [7].

Currently there are a number of estimates of $\kappa$ at the physical point, mostly based on the Taylor expansion method $[8,9,10]$, making use of different discretizations and observables. Another recent determination [11] has been obtained with analytic continuation and adopting a HISQ/tree action, in which the strange quark chemical potential $\mu_{s}$ is set equal to the light quark chemical potential $\mu_{l}$. We will later compare our findings with the ones in these other works (see Fig. 2). In this work we present a study [12] of the critical line at the physical point on the $N_{f}=2+1$ theory, performed using the analytic continuation method, which makes a reliable determination of

\footnotetext{
${ }^{1}$ For simplicity we will refer to the crossover as the "transition", even if no critical behavior is associated with it.
} 
$T_{c}\left(\mu_{I, B}\right)$ possible. We look at two observables, namely the renormalized chiral condensate and the renormalized chiral susceptibility, and for both quantities we obtain estimates of $T_{C}$ as a function of $\mu_{B}$ and thus of the curvature $\kappa$. We mainly study the situation in which $\mu_{s}=0$, but also check the case in which $\mu_{s}=\mu_{l}$.

The analytic continuation approach relies on the fact that if we choose $\mu$ to be on the imaginary axis $\left(\mu=i \mu_{i}\right)$, the sign problem is non existent. By choosing $\mu_{B}$ purely imaginary, equation (1.2) becomes

$$
\frac{T\left(\mu_{I, B}\right)}{T_{C}}=1+\kappa\left(\frac{\mu_{I, B}}{T\left(\mu_{I, B}\right)}\right)^{2}+O\left(\mu_{B}^{4}\right) .
$$

\section{Observables}

In order to evaluate the (pseudo) critical temperature, a definition of it is needed first. We do this by mean of two observables, namely the renormalized chiral condensate and the renormalized chiral susceptibility.

For the renormalized chiral condensate, we start from the chiral condensate for a generic flavour $f$ on the lattice, which is defined by

$$
\langle\bar{\psi} \psi\rangle_{l}=\frac{1}{V_{4}} \frac{\partial \log Z}{\partial m_{l}}=\frac{N_{l}}{4 V_{4}}\left\langle\operatorname{Tr} M_{l}^{-1}\right\rangle
$$

where $N_{f}$ is the degeneracy of the flavour $f$ and $M_{f}$ is the Dirac matrix with the $f$ quark mass. We consider the light quarks chiral condensate and choose its renormalization as in [13]:

$$
\langle\bar{\psi} \psi\rangle_{l}^{r}(T) \equiv \frac{\langle\bar{\psi} \psi\rangle_{l}(T)-\frac{2 m_{l}}{m_{s}}\langle\bar{\psi} \psi\rangle_{s}(T)}{\langle\bar{\psi} \psi\rangle_{l}(0)-\frac{2 m_{l}}{m_{s}}\langle\bar{\psi} \psi\rangle_{s}(0)}
$$

where $\langle\bar{\psi} \psi\rangle_{l} \equiv\langle\bar{\psi} \psi\rangle_{u}+\langle\bar{\psi} \psi\rangle_{d}$ represents the chiral condensate (2.1) for the light quarks, while $\langle\bar{\psi} \psi\rangle_{s}$ is the chiral condensate for the strange quark. The ratio is intended to be calculated between quantities estimated at the same lattice spacing.

For the renormalized chiral susceptibility, we start from the definition of the bare chiral susceptibility on the lattice

$$
\chi_{\bar{\psi} \psi} \equiv \frac{\partial^{2} \log Z}{\partial m_{l}^{2}}=\frac{1}{V_{4}}\left(\frac{N_{l}}{4}\right)^{2}\left\langle\left(\operatorname{Tr} M_{l}^{-1}\right)^{2}\right\rangle-\frac{1}{V_{4}}\left(\frac{N_{l}}{4}\right)^{2}\left\langle\operatorname{Tr} M_{l}^{-1}\right\rangle^{2}+-\frac{1}{V_{4}} \frac{N_{l}}{4}\left\langle\operatorname{Tr} M_{l}^{-2}\right\rangle,
$$

where $N_{l}$ is the number of light flavours in the theory and $M_{l}$ is the Dirac matrix with light quarks mass. We have chosen to renormalize it as described in [2], namely as

$$
\chi_{\bar{\psi} \psi}^{r}(T) \equiv m_{l}^{2}\left(\chi_{\bar{\psi} \psi}(T)-\chi_{\bar{\psi} \psi}(0)\right)
$$

where the factor $m_{l}^{2}$ removes the multiplicative UV divergences, while by subtracting the value of the susceptibility at zero temperature the additive UV divergences are eliminated. In the plots and for the fits we use the dimensionless quantity $\chi_{\bar{\psi} \psi}^{r}(T) / m_{\pi}^{4}$. 


\section{Numerical Setup}

We simulated $N_{f}=2+1$ QCD with physical quark masses, using the stout smeared staggered fermions formulation for the quark fields, and a tree level Symanzik improved action for the gauge part. Our simulation parameters for the coupling $\beta$ and the bare quark masses were obtained interpolating the data reported in $[14,15]$ in order to move on a line of constant physics with $m_{\pi} \equiv 135 \mathrm{MeV}$ and $m_{s} / m_{l}=28.15$.

In order to apply our renormalization procedure we need to evaluate both $\langle\bar{\psi} \psi\rangle_{f}$ and $\chi_{\bar{\psi} \psi}$ at zero temperature, thus we measured the necessary quantities on a $32^{4}$ lattice for a selection of $\beta$ values, and then interpolate the results.

We have acquired data on $16^{3} \times 6,24^{3} \times 6,32^{3} \times 6$ and $32^{3} \times 8$ lattices for both renormalized observables, for a few values of the chemical potential of the light quarks $\mu_{l}$, with the strange quark chemical potential $\mu_{s}$ set to zero. On the $32^{3} \times 8$ lattice we also studied the setup in which $\mu_{s}=\mu_{l}$, in order to test the dependence of the results on $\mu_{s}$. For each value of $\mu_{l}$ we have performed simulations for $\mathscr{O}(10)$ values of $T$ located around the transition, and the data for both observables has been obtained with noisy estimators, with 8 random vectors for each flavour.

Our simulations were run on the Blue Gene/Q Fermi at CINECA and on the CNS4 Zefiro cluster at INFN-Pisa.

\section{Numerical results}

Since the rapid change of properties that we are studying is not a real phase transition, even in the thermodynamic limit the value of the critical temperature depends on the definition of the critical temperature itself. The behaviour of the renormalized chiral condensate $\langle\bar{\psi} \psi\rangle_{l}^{r}$ with the temperature $T$ at fixed $\mu_{B}$ is well described, within our the statistical uncertainties, by an arctangent-like function (see left column of Fig.1). In order to define $T_{c}\left(\mu_{B}\right)$, we performed a best fit of the data with the function

$$
\langle\bar{\psi} \psi\rangle_{l}^{r}(T)=A_{1}+B_{1} \arctan \left(C_{1}\left(T-T_{c}\right)\right),
$$

so that $T_{c}\left(\mu_{B}\right)$ is defined as the inflection point of $\langle\bar{\psi} \psi\rangle_{l}^{r}(T)$ (see left column of Fig. 1). Function (4.1) turns out to well describe the data on the whole range of explored temperatures. We evaluated the systematic error on $T_{c}\left(\mu_{B}\right)$ by varying the chosen interpolation (to a rational function or an hyperbolic tangent), while the statistical error on the fit parameters were evaluated through a bootstrap technique.

As far as the renormalized chiral susceptibility is concerned, in order to give an estimate of the critical temperature we performed a fit of the dimensionless quantity $\chi_{\bar{\psi} \psi}^{r}(T) / m_{\pi}^{4}$ with a Lorentzian function, which describes reasonably well the data around the peak:

$$
\chi_{\bar{\psi} \psi}^{r}=\frac{A}{B^{2}+\left(T-T_{c}\left(\mu_{B}\right)\right)^{2}} .
$$

The critical temperature obtained from $\chi_{\bar{\psi}}^{r}$ is defined as the location of its maximum (see right column of Fig.1). The systematic error were estimated by assessing the dependence of $T_{c}\left(\mu_{B}\right)$ on the choice of the fitting range, while the statistical error was determined by a bootstrap technique. We fitted the $T_{c}(\mu)$ data according to (1.2), in order to find the curvature $\kappa$ and the critical temperature 

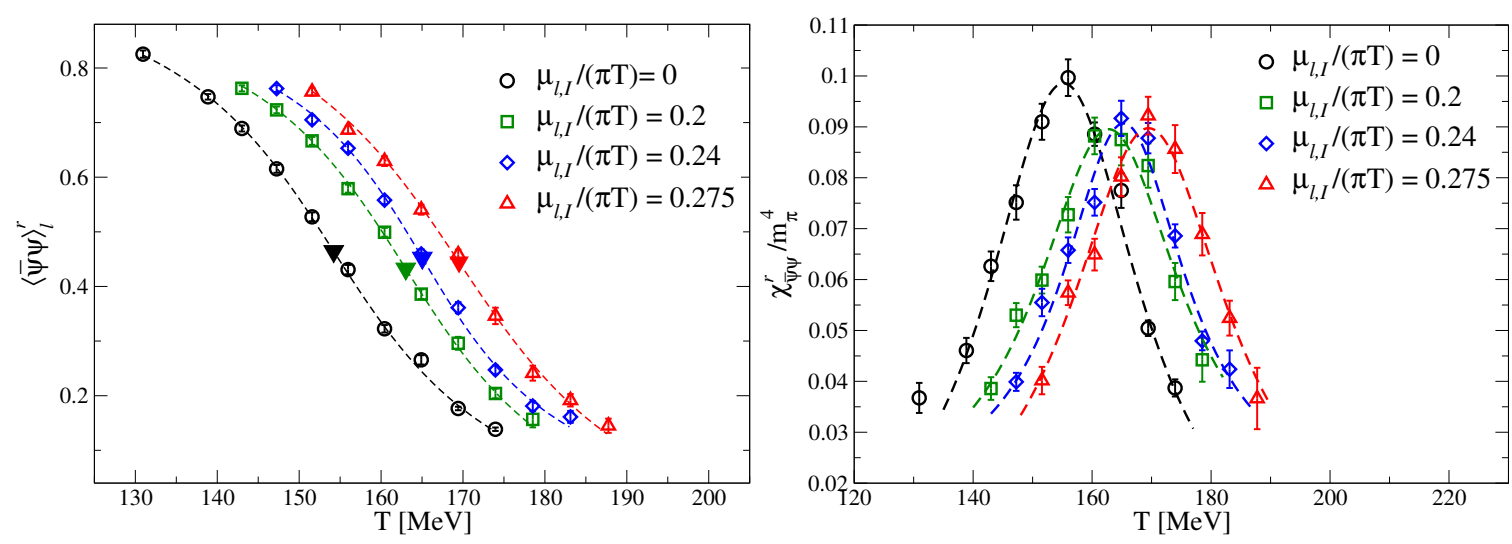

Figure 1: Data for both observables, with the curves of the best fit used to determine $T_{c}(\mu)$ (results from the $32^{3} \times 8$ lattice with $\mu_{s}=0$ ). The results of the best fits, in the form of 4.1 and 4.2 are listed in [12]. Left : Renormalized light chiral condensate (for the sake of readability, the values at the critical temperature are denoted with filled triangles); Right : Renormalized light chiral susceptibility divided by $m_{\pi}^{4}$; Lines correspond to the best fit described in the text and the filled triangles denote the values at the pseudo-critical temperature.

at zero chemical potential $T_{c}$. While in the $\mu_{s}=0$ setup the quadratic form is sufficient to well describe the data, in the case of the $\mu_{s} \neq 0$ setup it is necessary to add a quartic term in $\mu_{B}$ to have a reasonable value of $\chi^{2}$. In fact, the introduction of a non-zero strange quark chemical potential causes an increase of the relative temperature change $T_{c}\left(\mu_{B}\right) / T_{c}-1$ which is up to $40 \%$ larger than in the $\mu_{s}=0$ case (see Fig.2). It is tempting to put this aspect in relation to the fact that, in the $\mu_{s}=\mu_{l}$ setup, the Roberge-Weiss endpoint is closer to the $\mu_{l}=0$ axis, thus enhancing non-linear corrections (for a detailed discussion of this point, and of the effect of $\mu_{s}$ on the Roberge-Weiss transition, see [12]). When comparing the $T_{c}\left(\mu_{I, B}\right)$ curves obtained for the $\mu_{s}=0$ and $\mu_{s}=\mu_{l}$ cases from the $32^{3} \times 8$ lattice, the coefficients of the quadratic term (which are proportional to the curvatures) are well compatible within uncertainties if we include quartic corrections in the latter situation.

By using the data from the $24^{3} \times 6$ and $32^{3} \times 8$ lattices and assuming that the finite cut-off effects are of order $a^{2} \propto 1 / N_{t}^{2}$, we can try a rudimentary continuum scaling analysis for the curvature. We obtain $\kappa=0.0132(18)$ from the renormalized chiral condensate and $\kappa=0.0126(22)$ from the renormalized chiral susceptibility. An assessment of the finite size effects is done in [12].

Our estimates of $\kappa$ can be compared with the results of a selection of recent works which look at the chiral transition in $N_{f}=2+1 \mathrm{QCD}$, at or near the physical point $[8,9,11]$. Our result seem generally larger than those obtained by the Taylor expansion method, and in marginal agreement with the result obtained by analytic continuation but with a different discretization. A comparison between the results of these works and ours is shown in the right side of Fig. 2. However, a correct assessment of the discrepancies and agreements between our results and the ones of other works requires a careful analysis of all the possible sources of systematic differences between the various determinations. While lattice discretization effects should vanish in the continuum limit, there is a possible discrepancy due to the ambiguities in the definition of the critical temperature, as already stated. A discussion of these aspects is reported in [12]. 

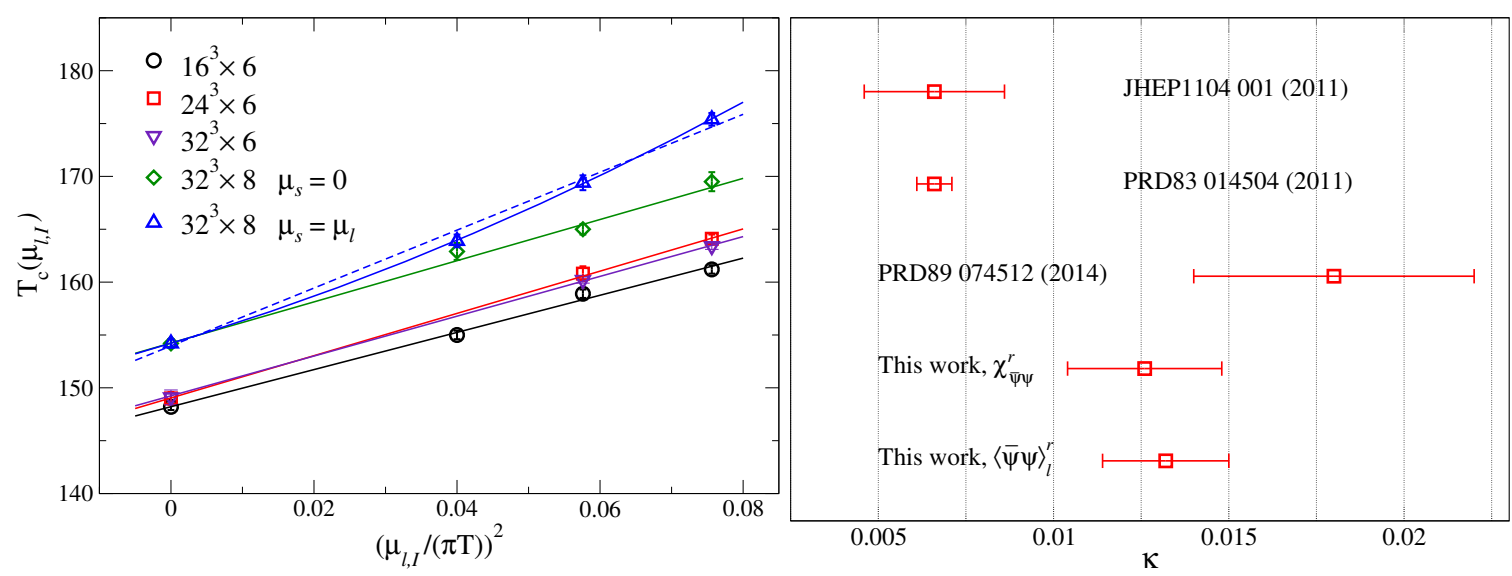

Figure 2: Left: Estimates of $T_{c}$ obtained from the renormalized chiral condensate for various values of the chemical potential and lattice sizes. The lines correspond to quadratic or quartic fits in $\mu_{l, I}$, as discussed in the text. The value of the parameters obtained by the best fit, and the corresponding graph for the renormalized chiral susceptibility can be found in [12]. Right: Determinations of the critical line curvature $\kappa$ in a selection of recent works, which can be compared to ours. From bottom to top: $i$ ) renormalized chiral condensate, this work; ii) renormalized chiral susceptibility, this work; iii) analytic continuation, disconnected chiral susceptibility with $\mu_{s}=\mu_{l}$ [11]; iv) Taylor expansion, chiral susceptibility [8]; v) Taylor expansion, chiral condensate (different renormalization) [9].

\section{Conclusions}

We presented a determination of the pseudo-critical line of $N_{f}=2+1$ QCD with physical quark masses by the method of analytic continuation from an imaginary chemical potential. We considered a stout smeared staggered discretization and performed simulations on lattices with $N_{t}=6$ and $N_{t}=8$ at the physical point. In order to locate the pseudo-critical temperature $T_{c}$, we have considered both the inflection point of the renormalized chiral condensate and the location of the peak of the renormalized chiral susceptibility. The pseudo-critical temperature at zero quark chemical potential is found to be in agreement with previous determinations. A preliminary continuum scaling analysis of the curvature yields $\kappa=0.0132(18)$ for the chiral condensate and $\kappa=0.0126(22)$ for the chiral susceptibility. In the future we plan to extend our investigation to lattices with at least $N_{t}=10$, in order to assess the systematics associated with the continuum extrapolation.

Since the value of the pseudo-critical temperature depends on its definition, at least part of the differences between the different determinations of the curvature of the critical line present in the literature can be explained by a careful analysis of the procedures used to obtain it. For example, if we analyse our data following as much as possible the methods in [9] or in [11], we obtain determinations of $\kappa$ which compare better to the results of the respective works ${ }^{2}$ (see [12]).

We have also considered the case of $\mu_{s}=\mu_{l}$. The quadratic term in the parametrization of the critical line does not change significantly, but non-linear terms in $\mu_{l}^{2}$ become evident. It is suggested in [12] that the origin of this difference could be related to the fact that, in this case, the

\footnotetext{
${ }^{2}$ The present work and [9] share the same discretization, but there the Taylor expansion method is used. In [11] the discretization is different from the one adopted in the present work, together with analytic continuation.
} 
so-called Roberge-Weiss line and the associated non-analytic behaviour moves closer to the $\mu_{l}=0$ axis.

\section{References}

[1] Y. Aoki, Z. Fodor, S. D. Katz and K. K. Szabo, Phys. Lett. B 643, 46 (2006). S. Borsanyi et al., JHEP 1009, 073 (2010). A. Bazavov, T. Bhattacharya, M. Cheng, C. DeTar, H. T. Ding, S. Gottlieb, R. Gupta and P. Hegde et al., Phys. Rev. D 85, 054503 (2012). T. Bhattacharya, M. I. Buchoff, N. H. Christ, H. -T. Ding, R. Gupta, C. Jung, F. Karsch and Z. Lin et al., Phys. Rev. Lett. 113, 082001 (2014).

[2] Y. Aoki, G. Endrodi, Z. Fodor, S. D. Katz and K. K. Szabo, Nature 443 (2006) 675.

[3] P. de Forcrand and O. Philipsen, Nucl. Phys. B 642, 290 (2002); Nucl. Phys. B 673, 170 (2003). M. D’Elia and M. P. Lombardo, Phys. Rev. D 67, 014505 (2003), Phys. Rev. D 70, 074509 (2004).

[4] E. Laermann, F. Meyer and M. P. Lombardo, J. Phys. Conf. Ser. 432, 012016 (2013). E. Laermann, F. Meyer and M. P. Lombardo, arXiv:1304.3247 [hep-lat].

[5] P. Braun-Munzinger, J. Stachel, J. P. Wessels and N. Xu, Phys. Lett. B 344, 43 (1995); Phys. Lett. B 365, 1 (1996). F. Becattini, Z. Phys. C 69, 485 (1996). F. Becattini and U. W. Heinz, Z. Phys. C 76, 269 (1997), [Erratum-ibid. C 76, 578 (1997)]; F. Becattini, J. Cleymans, A. Keranen, E. Suhonen and K. Redlich, Phys. Rev. C 64, 024901 (2001). P. Braun-Munzinger, D. Magestro, K. Redlich and J. Stachel, Phys. Lett. B 518, 41 (2001). A. Andronic, P. Braun-Munzinger and J. Stachel, Nucl. Phys. A 772, 167 (2006).

[6] J. Cleymans, H. Oeschler, K. Redlich and S. Wheaton, Phys. Rev. C 73, 034905 (2006).

[7] F. Becattini, M. Bleicher, T. Kollegger, T. Schuster, J. Steinheimer and R. Stock, Phys. Rev. Lett. 111, 082302 (2013).

[8] O. Kaczmarek, F. Karsch, E. Laermann, C. Miao, S. Mukherjee, P. Petreczky, C. Schmidt, W. Soeldner and W. Unger, Phys. Rev. D 83, 014504 (2011).

[9] G. Endrodi, Z. Fodor, S. D. Katz and K. K. Szabo, JHEP 1104, 001 (2011).

[10] S. Borsanyi, G. Endrodi, Z. Fodor, S. D. Katz, S. Krieg, C. Ratti and K. K. Szabo, JHEP 1208, 053 (2012).

[11] P. Cea, L. Cosmai and A. Papa, Phys. Rev. D 89, 074512 (2014).

[12] C. Bonati, M. D’Elia, M. Mariti, M. Mesiti, F. Negro, F. Sanfilippo Phys. Rev. D 90, 114025 (2014).

[13] M. Cheng, N. H. Christ, S. Datta, J. van der Heide, C. Jung, F. Karsch, O. Kaczmarek and E. Laermann et al., Phys. Rev. D 77, 014511 (2008).

[14] Y. Aoki, S. Borsanyi, S. Durr, Z. Fodor, S. D. Katz, S. Krieg and K. K. Szabo, JHEP 0906, 088 (2009).

[15] S. Borsanyi, G. Endrodi, Z. Fodor, A. Jakovac, S. D. Katz, S. Krieg, C. Ratti and K. K. Szabo, JHEP 1011, 077 (2010). S. Borsanyi, Z. Fodor, C. Hoelbling, S. D. Katz, S. Krieg and K. K. Szabo, Phys. Lett. B 730, 99 (2014). 